\title{
DINÁMICA DE LA INFECCIÓN CON Mycoplasma hyopneumoniae EN PORCINOS PROVENIENTES DE MADRES CON Y SIN ANTECEDENTES DE INMUNIZACIÓN
}

\author{
Vanessa Bachmann C. ${ }^{1}$, Sonia Calle E. ${ }^{2,3}$, Marlon Torres A. ${ }^{4}$, César Gavidia Ch. ${ }^{5}$, \\ Siever Morales C. ${ }^{2}$ y Francisco Acosta C. ${ }^{1}$
}

\section{Abstract}

The aim of the study was to determine the time of infection with Mycoplasma hyopneumoniae in 240 piglets of vaccinated and non vaccinated sows from a positive farm with an all-in/all-out (AIAO) system, located in the Chillón valley, Lima. The study was conducted during autumn and winter 2003. One group of sows was vaccinated with $2 \mathrm{ml}$ of a $M$. hyopneumoniae commercial bacterin at 85 days of gestation while the other group remained unvaccinated. Two groups of 120 piglets each were established according to their dams' status of immunity. Blood samples were collected from piglets at various times during the production period (weaning till slaughter) for measuring antibodies against $M$. hyopneumoniae using an ELISA indirect assay. The $66.6(74 / 112)$ and $85.6 \%$ (101/118) of piglets from the vaccinated and non-vaccinated dams, respectively, were seroreactive throughout the period of the study. The results indicated that the time of infection occurred in both groups at approximately 10 weeks of age, and without significant differences between groups ( $>0.05$ ). Time of infection occurred concomitantly with the stress caused by the relocation of piglets from nursery to growth-finishing corrals.

Key words: Mycoplasma hyopneumoniae, pig, seroconversion, seroreactive

\section{Resumen}

El presente estudio tuvo por objetivo determinar el momento de infección con Mycoplasma hyopneumoniae en 240 porcinos provenientes de madres inmunizadas y no inmunizadas, en una granja tecnificada de un solo sitio, donde se maneja un sistema de "todo dentro todo fuera". La granja estaba ubicada en el valle del río Chillón, Lima, y era positiva a este agente. El estudio se realizó en los meses de otoño e invierno del 2003. Un grupo de marranas fue inmunizado con $2 \mathrm{ml}$ de una bacterina comercial contra $M$. hyopneumoniae a los 85 días de gestación, mientras que otro grupo permaneció sin vacunación. Los lechones obtenidos de estas marranas fueron divididos en dos grupos de 120 animales, según los antecedentes inmunitarios de las madres. Se obtuvieron muestras de suero de los porcinos de ambos grupos en diferentes momentos del proceso

\footnotetext{
${ }^{1}$ Práctica privada

${ }^{2}$ Laboratorio de Microbiología y Parasitología Veterinaria, FMV-UNMSM

${ }^{3}$ E-mail: calleson@ gmail.com

${ }^{4}$ Laboratorio de Producción Agropecuaria, FMV-UNMSM

${ }^{5}$ Laboratorio de Epidemiología Veterinaria Preventiva, FMV-UNMSM
} 
productivo para la medición de anticuerpos mediante la prueba de ELISA indirecta. El 66.7 (74/112) y $85.6 \%$ (101/118) de animales provenientes de madres inmunizadas y no inmunizadas, respectivamente, fueron serorreactores durante el transcurso del estudio. Los resultados obtenidos sugieren que la infección de ambos grupos ocurrió alrededor de las 10 semanas de edad, sin diferencia estadística significativa entre grupos $(\mathrm{p}>0.05)$ lo cual coincide con factores estresantes como fue el reagrupamiento de los animales de los corrales de recría a los de acabado.

Palabras clave: Mycoplasma hyopneumoniae, porcino, seroconversión, serorreactor

\section{INTRODUCCIÓN}

La neumonía causada por el $\mathrm{Myco}$ plasma hyopneumoniae afecta específicamente a los porcinos. Se presenta una mayor incidencia en granjas de crianza intensiva, afectando el rendimiento productivo y causando grandes pérdidas económicas (Ross, 1986; Blood y Radostits, 1992). Es una enfermedad de alta morbilidad y baja mortalidad que afecta principalmente a animales en crecimiento (Sheidt et al., 1994). Se transmite por contacto directo con secreciones respiratorias de las madres o compañeros de corral que se encuentran infectados (Ross, 1999), o por vía aerosol (Burch, 2003a).

El germen coloniza tráquea, bronquios y bronquiolos, produciendo cilioestasis $\mathrm{e}$ inmunosupresión, debilitando la primera barrera mecánica de defensa del sistema respiratorio, y permitiendo la entrada de agentes secundarios, agravando el cuadro y las pérdidas económicas (Thacker et al., 1998).

Estudios serológicos realizados en Lima indican la presencia de este agente en granjas tecnificadas (Huallanca, 1999) y de crianza artesanal (Ibarra, 2000); sin embargo, la mayoría de granjas porcinas del país no vacuna ni toma medidas preventivas contra este agente.

Diversos estudios realizados en el extranjero han tenido por objetivo determinar el momento óptimo de vacunación, así como evaluar el efecto de la bacterina contra $M$. hyopneumoniae sobre el rendimiento productivo (Burch, 2003b; Clark, 1999; Scheidt et al.,1994). Los resultados obtenidos son mayormente aplicables a la granja o sistema de producción donde se realizó el estudio, debido a que los niveles inmunitarios transferidos de madres a lechones y los factores medio ambientales varían de una granja a otra. Es por esto, que el objetivo del presente estudio fue determinar el momento de infección con Mycoplasma hyopneumoniae en porcinos provenientes de madres con y sin inmunización contra este agente, con el fin de tener un precedente serológico local e incentivar a otras granjas de realizar trabajos similares para determinar el calendario de vacunación respectivo.

\section{Materiales y Métodos}

\section{Lugar de estudio}

El trabajo se llevó a cabo durante los meses de otoño e invierno del 2003 en una granja porcina tecnificada, positiva a $M$. hyopneumoniae, ubicada en el valle del río Chillón, provincia y departamento de Lima. Las muestras de suero colectadas fueron procesadas en el Laboratorio de Bacteriología de la Facultad de Medicina Veterinaria de la Universidad Nacional Mayor de San Marcos.

\section{Sistema de manejo}

La granja porcina cuenta con un plantel de 600 madres reproductoras donde no se practica la inmunización contra M. hyopneu- 
moniae ni emplea antibióticos micoplasmicidas como medida de control. La granja maneja un sistema de "todo dentro todo fuera".

Los lechones permanecen con sus madres por un periodo de $19 \pm 2$ días, y luego del destete se les traslada a jaulas de recría, donde permanecen hasta los 70 días de edad. De allí son llevados a los corrales de engorde donde permanecen hasta los 148 días de edad, en que alcanzan el peso al mercado $(90 \mathrm{~kg})$.

La granja dispone de un sistema estricto de bioseguridad. Los vehículos son lavados y desinfectados antes de su ingreso. En la entrada de cada área se encuentran pediluvios con desinfectante. Los trabajadores y visitantes se bañan antes de ingresar a las instalaciones y emplean un vestuario proporcionado por la granja.

\section{Animales}

Se empleó un tamaño muestral de 240 animales. Se utilizó un grupo de 120 lechones provenientes de madres inmunizadas (MI) contra M. hyopneumoniae y 120 provenientes de madres no inmunizadas (MNI). Las madres eran multíparas y los lechones fueron producto de un cruce comercial. Para el estudio, se seleccionaron 28 marranas programadas para parir dentro de la misma semana. La mitad fue inmunizada con $2 \mathrm{ml}$ de una bacterina comercial contra $M$. hyopneumoniae a los 85 días de gestación y las restantes permanecieron sin inmunizar. Los grupos de lechones fueron formados al momento del destete y compartieron el mismo ambiente durante el transcurso del estudio. Los lechones fueron identificados con tatuajes en la oreja a fin de realizar el seguimiento individual de cada animal.

\section{Método de recolección y procesamiento de muestras}

Las muestras de sangre fueron obtenidas por punción de la vena cava, con sistema de tubo al vacío, a los 21, 39, 70, 84, 112 y 148 días de edad. Las muestras se procesaron con un kit comercial de ELISA indirecto
(HerdCheck*M hyo). El espectrofo-tómetro se calibró con un nivel de absorbancia de $650 \mathrm{~nm}$.

La prueba de ELISA indirecta define como positivo a las muestras con coeficientes de muestras positivas (M/P) mayores o iguales al 0.4 , negativo si la relación $\mathrm{M} / \mathrm{P}$ es menor o igual al 0.3 y sospechoso si la muestra se encuentra entre 0.3 y 0.4 .

\section{Análisis estadístico}

El estado inmunitario de los cerdos provenientes de madres con y sin inmunización al inicio del estudio se analizó por medio de la prueba de " $\mathrm{t}$ " Student independiente. El grado de asociación entre el incremento en los títulos de anticuerpos durante el estudio y la condición inmunitaria de la madre se evaluó con la prueba de ChiCuadrado.

Se determinó la curva de tendencia y la edad en la que los animales fueron serorreactores. Los datos se analizaron mediante una curva de supervivencia empleando la prueba de Kaplan Meier. Se determinó si existía diferencia estadística en el momento de seroconversión entre los individuos provenientes de MI y MNI mediante la prueba de Log-Rank con nivel de confianza del $95 \%$.

\section{Resultados y Discusión}

El 31.7\% (38/120) de los lechones provenientes de MNI resultaron positivos a la lectura de la prueba de ELISA indirecta, frente al $85.8 \%(103 / 120)$ de los animales nacidos de MI (Cuadro 1, p< 0.01). Esta diferencia era de esperarse, ya que los lechones que provienen de MI tienen un nivel de inmunoglobulinas maternas de hasta tres veces mayor que los que provienen de MNI (Clark, 1999).

Los animales provenientes de MI permanecieron con un nivel alto de anticuerpos maternales hasta los 39 días de edad, mien- 
Cuadro 1. Frecuencia de lechones seropositivos a Mycoplasma hyopneumoniae en la prueba de ELISA Indirecta, según la edad y condicion inmunitaria de las madres

\begin{tabular}{|c|c|c|c|c|c|c|}
\hline \multirow{2}{*}{$\begin{array}{l}\text { Edad } \\
\text { (días) }\end{array}$} & \multicolumn{3}{|c|}{ Madres Inmunizadas } & \multicolumn{3}{|c|}{ Madres No Inmunizadas } \\
\hline & $\mathrm{N}^{\mathrm{o}}$ & Positivos & $\%$ & $\mathrm{~N}^{\mathrm{o}}$ & Positivos & $\%$ \\
\hline 21 & 120 & 103 & 85.8 & 120 & 38 & 31.7 \\
\hline 39 & 120 & 81 & 67.5 & 120 & 10 & 8.3 \\
\hline 70 & 120 & 22 & 18.3 & 119 & 6 & 5.0 \\
\hline 84 & 119 & 11 & 9.2 & 118 & 8 & 6.8 \\
\hline 112 & 117 & 36 & 30.8 & 118 & 33 & 28.0 \\
\hline 148 & 112 & 60 & 53.6 & 118 & 92 & 78.0 \\
\hline
\end{tabular}

tras que los animales provenientes de MNI permanecieron con anticuerpos elevados hasta los 21 días de edad (Cuadro 1), lo cual coincide con otros reportes (Burch, 2003b).

Se tuvo la pérdida de 8 animales provenientes de MI y de 2 animales de MNI durante el desarrollo del trabajo, debido a problemas digestivos ajenos al estudio; sin embargo, ésto no afectó el análisis final de los resultados, ya que los cálculos se hicieron en base a los animales vivos.

El porcentaje de serorreactores incrementó con la edad de los animales en ambos grupos (Cuadro 2). La Prueba de Log-Rank demostró que no hubo diferencia estadística significativa en el momento de seroconversión entre los individuos provenientes de MI y MNI ( $>0.05$ ).

Otros estudios demuestran que la seroconversión ocurre entre las 10 y 16 semanas de edad (Suh et al., 1998) y el mayor índice de seroconversión se produce entre las 12 y 16 semanas de edad (Blood et al., 1999). Así mismo, la seroconversión se presenta entre 2 a 8 semanas después de la infección experimental (Thacker, 2001). Tomando en cuenta esto, el periodo crítico en que ocurrió la infección natural para los animales provenientes de MI fue entre las 10 y 12 semanas de edad, ya que éstos resultaron serorreactores a las 12 a 16 semanas. Este momento coincide con el final de la etapa de recría y el reagrupamiento de animales en los corrales de engorde, periodo muy estresante para los animales. Por otro lado, el periodo crítico de infección para los lechones que provienen de MNI fue entre las 10 y 14 semanas de edad. Estos hallazgos coinciden con Leon et al. (2001), quienes aseveran que el periodo crítico para transmisión de la enfermedad se da al inicio de la etapa de acabado, cuando disminuye la concentración de anticuerpos maternales.

El $66.1(74 / 112)$ y $85.6 \%$ (101/118) de animales que provienen de MI y MNI, respectivamente, seroconvirtieron en el transcurso del estudio (Cuadro 3). Torres et al. (2003) demostró que, a pesar de que el $M$. hyopneumoniae se encuentra presente en el tracto respiratorio de los animales durante todo el periodo productivo, los animales comienzan a seroconvertir luego que desciende el nivel de anticuerpos maternales en la mayoría de los animales de la población.

En el presente estudio se esperaba encontrar que los lechones del grupo de animales que procedían de MNI se infectaran antes que los animales provenientes de MI, debido a la mayor susceptibilidad a la infección 
Cuadro 2. Número y porcentaje de lechones que seroconvirtieron a Mycoplasma hyopneumoniae, según condición inmunitaria de las madres y la edad del lechón

\begin{tabular}{|c|c|c|c|c|c|c|}
\hline \multirow{2}{*}{$\begin{array}{l}\text { Edad } \\
\text { (días) }\end{array}$} & \multicolumn{3}{|c|}{ Madres inmunizadas } & \multicolumn{3}{|c|}{ Madres no inmunizadas } \\
\hline & $\mathrm{N}^{\mathrm{o}}$ & Positivos & $\%$ & $\mathrm{~N}^{\mathrm{o}}$ & Positivos & $\%$ \\
\hline 39 & 120 & 1 & 0.8 & 120 & 3 & 2.5 \\
\hline 70 & 120 & 3 & 2.5 & 119 & 5 & 4.2 \\
\hline 84 & 119 & 5 & 4.2 & 118 & 6 & 5.1 \\
\hline 112 & 117 & 34 & 29.1 & 118 & 25 & 21.2 \\
\hline 148 & 112 & 31 & 27.7 & 118 & 62 & 53.0 \\
\hline
\end{tabular}

Cuadro 3. Número y porcentaje de animales seroreactores a M. hyopneumoniae en el transcurso del estudio, según condición inmunitaria de las madres y la edad del lechón

\begin{tabular}{ccccccc}
\hline \multirow{2}{*}{$\begin{array}{c}\text { Condición inmunitaria } \\
\text { de los cerdos }\end{array}$} & \multicolumn{2}{c}{ Positivos } & & \multicolumn{2}{c}{ Negativos } \\
\cline { 2 - 3 } \cline { 5 - 6 } \cline { 5 - 6 } \cline { 5 - 6 } & Número & Porcentaje & & Número & Porcentaje \\
\hline Madres inmunizadas & 74 & 66.1 & & 38 & 33.9 \\
Madres no inmunizadas & 101 & 85.6 & & 17 & 14.4 \\
\hline
\end{tabular}

por ausencia de anticuerpos maternales. Sin embargo, a pesar de los hallazgos encontrados, no se encontró diferencia significativa, ya que ambos grupos comenzaron a seroconvertir al inicio de la etapa de acabado (10 semanas de edad). La coincidencia en el momento de seroconversión en los animales de ambos grupos puede deberse a que existe un efecto de la inmunidad materna del grupo de animales que proviene de MI sobre la inmunidad de masa, ya que mientras los anticuerpos maternales del grupo de MI se mantiene alto, la seroconversión de toda la población se ve retrasada, pero una vez que los anticuerpos maternales de éstos bajan, la presión del agente se hace mayor y ambos grupos de animales comienzan a seroconvertir.
También puede deberse a que se necesita una dosis infectiva bastante alta para que los animales comiencen a seroconvertir y ésta pueda ir haciéndose cada vez mayor conforme los animales vayan perdiendo la inmunidad materna y el agente comience a reproducirse en el tracto respiratorio, incrementando el número de microorganismos en el ambiente y facilitando la infección de la piara. Esto se sustenta en lo mencionado por Thacker (2001), quien afirma que se desconoce lo que induce a la seroconversión, pero es probable que ocurra cuando el número de microorganismos alcanzan un periodo crítico frente al sistema inmune, resultando en la formación de anticuerpos. De la misma manera, se sustenta en lo afirmado por Wallgren et al. (1998), quienes aseveran que la edad 
en que los cerdos se infectan, depende del balance entre las inmunoglobulinas protectivas provenientes del calostro maternal y la carga patógena de la piara.

El incremento del número de microorganismos en la piara se vió favorecido por la época del año en que se realizó el estudio, ya que el frío y el alto porcentaje de humedad favorecen la diseminación de la enfermedad (Burch, 2003a; Scheidt et al., 1990).

\section{Conclusiones}

- No se encontró diferencia significativa ( $p>0.05$ ) en el momento de seroconversión de los lechones provenientes de madres inmunizadas y no inmunizadas contra Mycoplasma hyopneumoniae.

- El momento de infección (10-12 semanas) ocurre durante la ausencia de anticuerpos maternales en la población de animales que procedían de madres inmunizadas y coincide con la presencia de factores estresantes externos (cambio de corrales).

- El momento óptimo de vacunación en la granja donde se realizo el estudio es a las 6 semanas de edad en animales que provienen de madres no inmunizadas y a las 9 semanas en los que proceden de madres inmunizadas.

\section{Literatura Citada}

1. Blood, D.C.; O. Radostits; K. Hinchellf. 1999. Manual de Medicina Veterinaria. Texto de las enfermedades del vacuno, porcino, caprino y equino. $9^{\mathrm{a}}$ ed. Vol. III. p 1195-1204. Ed. McGraw Hill. Madrid, España.

2. Blood, D.C.; O. Radostits. 1992. Manual de Medicina Veterinaria. Texto de las enfermedades del vacuno, porcino, caprino y equino. $7^{\mathrm{a}}$ ed. Vol. II. p 843846. Ed. McGraw-Hill. Madrid, España.

3. Burch, D. 2003a. Mycoplasma vaccination - One shot or two? ScheringPlough Animal Health UK. Pig World Magazine. Octagon Services Ltd, Old Winsor, Berks, United Kingdom. Disponible en: http://www.octagonservices.co.uk/articles/respiratory.htm

4. Burch, D. 2003b. Mycoplasma vaccines and passive immunity. The effects of maternally delivers antibodies and piglet age vaccinal response. Schering-Plough Animal Health UK. Pig World Magazine. Octagon services Ltd, Old Winsor, Berks. United Kingdom. Disponible en: http://www.octagonservices.co.uk/articles/ mycoplasma2.htm

5. Clark, K.L. 1999. M. hyopneumoniae: Serology/vaccinology. American Association of Swine Practitioners 50: 365369.

6. Huallanca, A.C. 1999. Determinación de reactores a $M$. hyopneumoniae en cerdos sacrificados en un camal frigorífico. Tesis de Médico Veterinario. Facultad de Medicina Veterinaria, Univ. Nacional Mayor de San Marcos. Lima. 32 p.

7. Ibarra, M.A. 2000. Evidencia de la presencia de Mycoplasma hyopneumoniae en cerdos provinentes de granjas de crianza artesanal del sur de Lima. Tesis de Medico Veterinario. Facultad de Medicina Veterinaria, Univ. Nacional Mayor de San Marcos. Lima. 46 p.

8. Leon, E.A.; F. Madec; N.M. Taylor; M. Kobisch. 2001. Seroepidemiology of Mycoplasma hyopneumoniae in pigs from farrow-to-finish farms. Vet. Microbiol. 78: 3331-3341.

9. Ross, R.F. 1999. Mycoplasmal diseases. En: Diseases of swine. $8^{\text {th }} \mathrm{ed}$. p 495-509. Straw, B. et al. (eds). Iowa State University Press. Ames, Iowa.

10. Ross, R.F. 1986. Mycoplasmal diseases. En: Disease of swine. $6^{\text {th }}$ ed. $p$ 469-475. Leman A. et al. (eds). Iowa State University Press. Ames, Iowa. 
11. Scheidt, A.; V. Mayrose; W. Van Alstine. 1994. The effects of vaccinating pigs for Mycoplasmal pneumonia in a swine herd affected by enzootic pneumonia. Swine Health Prod. 2: 7-11.

12. Scheidt, A.; V.B. Mayrose; M.A. Hill. 1990. Relationship of growth performance to pneumonia and atrophic rhinitis detected in pigs at slaughter. J. Am. Vet. Med. Assoc. 196: 881-884.

13. Suh, D.; S. Rutten; S. Dee; H. Soo Joo; C. Pijoan. 1998. Effect of nursery depopulation on the seroprevalence of Mycoplasma hyopneumoniae infection in nursery pigs. J. Swine Health Prod. 6: 151-155.

14. Thacker, E.L. 2001. Mycoplasma diagnosis and immunity. Proc. Am. Assoc. Swine Veterinarians. p 467-469. Ohio, USA.

15. Thacker, B.J.; T. Boettcher; T. Anderson; E. Thacker; T. Young. 1998. The influence of passive immunity on serological responses to Mycoplasma hyopneumoniae vaccination. Proc. $15^{\text {th }}$ IPVS Congress. p 350-358. Birmingham.

16. Torres, M. 2003. Determinación serológica de la infección con Mycoplasma hyopneumoniae en una granja de cerdos de crianza intensiva. Tesis de Médico Veterinario. Facultad de Medicina Veterinaria. Univ. Nacional Mayor de San Marcos. Lima. 51 p.

17. Torres, M.; S. Calle; C. Cerón; R. Zacarías; N. Falcón; M. Morales. 2003. Evaluación de la cinética de la infección con Mycoplasma hyopneumoniae en porcinos de crianza intensiva. III Congreso Nacional de Porcicultura y Expo Porcina. p 59-62. Lima.

18. Wallgren, P.; G. Bölske; S. Gustafsson; S. Mattsson; C. Fossum. 1998. Humoral immune response to Mycoplasma hyopneumoniae in sows and offspring following an outbreak of mycoplasmosis. Vet. Microb. 60: 193-205. 